\title{
Diseño y Validación de una Escala de Preparatividad Académica para la Educación Superior en Chile
}

\author{
María J. Baeza-Rivera ${ }^{(1)}$, Andrés Antivilo ${ }^{(2)}$ y Lucio E. Rehbein ${ }^{(1) *}$ \\ (1) Departamento de Psicología, Universidad de La Frontera, Casilla 54-D,Temuco, Chile. \\ (e-mail: lucio.rehbein@ufrontera.cl) \\ (2) Facultad de Ciencias Sociales, Departamento de Psicología, Universidad de Chile, Santiago, Chile.
}

${ }^{*}$ Autor a quien debe ser enviada la correspondencia

Recibido Nov. 20, 2015; Aceptado Ene. 21, 2016; Versión final Mar. 21, 2016, Publicado Ago. 2016

\begin{abstract}
Resumen
Uno de los principales problemas de la educación terciaria es la alta tasa de deserción universitaria. Los antecedentes dan cuenta que para permanecer y tener éxito en la universidad se necesitan habilidades transversales que el estudiante debió desarrollar antes y que hemos denominado preparatividad académica. El objetivo del estudio fue construir un instrumento de medición que permita identificar y medir los factores que subyacen a la preparatividad académica. Los participantes fueron 603 estudiantes de enseñanza secundaria y terciaria. Se realizaron análisis factoriales exploratorios y confirmatorios para identificar y comprobar la estructura factorial de la escala desarrollada, la cual quedó conformada por 67 ítems agrupados en 7 factores que explican el 59,6\% de la varianza. La disponibilidad de la propuesta, denominada Escala de Preparatividad Académica para la Educación Superior (EPAES), permitirá la medición oportuna de la preparatividad y la aplicación oportuna de acciones remediales. Esto contribuirá a aumentar la retención de los estudiantes y así disminuir las actuales tasas de deserción universitaria.
\end{abstract}

\section{Development and Validation of an Academic Preparedness Scale for Chilean Higher Education}

\begin{abstract}
One of the major problems in tertiary education comes from its high students' dropout rates. The literature on this matter indicates that to stay and succeed in college, students must have developed, prior to enrolling, a set of transversal abilities, which we have named 'academic preparedness'. The aim of the study was to construct a measuring instrument that would help identifying and measuring the specific array of factors that affect academic preparedness. Participants were 603 volunteer students from secondary and tertiary education. After performing the necessary exploratory and confirmatory factor analyses, the definitive scale resulted in 67 items, grouped in 7 factors that explained $59.6 \%$ of variance. The availability of the Academic Preparedness Scale for Higher Education (EPAES) will allow the timely assessment of preparedness and the implementation of opportune remedial actions. This will contribute to retain students and therefore, to reduce the current university dropout rates.
\end{abstract}

Keywords: college preparedness; student retention; college dropout; assessment scale 


\section{INTRODUCCION}

Entre 1991 y 2011, la cantidad de jóvenes chilenos con edades entre 18 y 24 años que se matricularon en la educación terciaria aumentó de 127.628 a 1.068 .263 estudiantes (Zapata, Tejeda y Rojas, 2011). Aun cuando la cobertura ha aumentado, la proporción de individuos con educación superior completa es relativamente pequeña (Canales y de los Ríos, 2007). En otras palabras, pese a que se inscribe una gran cantidad de estudiantes en los establecimientos de educación superior, sólo un porcentaje menor de ellos culmina sus estudios universitarios (Donoso y Schiefelbein, 2007).

El Centro de Microdatos del Departamento de Economía de la Universidad de Chile (2008) indica que, al término del primer año, en las universidades adscritas al Consejo de Rectores de las Universidades Chilenas (en adelante, $\mathrm{CRUCH}$ ), el $19 \%$ de los alumnos deserta, cifra que aumenta a un $22 \%$ en las universidades privadas sin Aporte Fiscal Directo. A medida que pasan los años las tasas de deserción aumentan. De este modo, sumados los dos primeros años, esta tasa en los estudiantes matriculados en todas las carreras de las universidades del $\mathrm{CRUCH}$ se aproxima al 25\% (Consejo Superior de Educación, 2008). Y para el tercer y cuarto años, la deserción aumenta hasta $39 \%$ y $42 \%$, respectivamente, siendo los hombres quienes más desertan a partir del segundo año (Centro de Microdatos, 2008). Resultados similares han sido reportados por el informe de retención de estudiantes del ingreso a carreras del Ministerio de Educación (MINEDUC, 2010).

Aun cuando los estudiantes que postulan a la Educación Superior cumplen con los requisitos de ingreso a la universidad, ello parece no implicar que se encuentren preparados para desarrollar el tipo de conductas y desempeños que son requeridos en ese nivel educacional. Por tanto, no estarían en condiciones de aprobar los cursos sin ser objeto de alguna intervención nivelatoria o remedial (Lombardi et al., 2011). Dicho de otra manera, los antecedentes recién expuestos sugieren una falta de alineamiento entre los contenidos y las competencias que los jóvenes aprenden en la Educación Media y las exigencias propias de cada carrera en la Educación Superior. Estas brechas (Centro Microdatos-Universidad de Chile, 2008) incluyen, entre otras, debilidades en contenidos, escasos hábitos de estudios, marcadas diferencias en las metodologías de enseñanza y aprendizaje de la universidad. Quienes se ven más perjudicados son los alumnos provenientes de establecimientos municipalizados y de escasos recursos, ya que ellos tendrían mayores debilidades académicas previas (Donoso, 2010).

Asimismo, es también relevante que la elección de carrera sea certera y guarde fidelidad con los intereses y competencias cognitivas, personales y sociales de los jóvenes. Una elección errónea significa una pérdida económica y de tiempo, además del costo emocional y la frustración tanto para los estudiantes como para sus familias (Donoso, 2010). La evidencia ya presentada permite afirmar que la deserción universitaria no depende exclusivamente de la calidad del establecimiento de educación superior; los indicadores de tasa de retención, tasa de aprobación y tasa de retraso, están influidos por muchas variables entre las que se incluyen aquellas determinadas por características de los alumnos y que, como tales, no pueden ser totalmente controladas por las instituciones (de los Ríos, 2000).

Astin (1984) plantea que el aprendizaje de los estudiantes y su retención en la universidad dependen del nivel de involucramiento y atracción que ésta ejerza en sus estudiantes. A su vez, el involucramiento depende de la relación entre los profesores y sus estudiantes, de la integración de estos últimos con sus pares, y de su participación en las actividades académicas, sociales, e institucionales (Tinto, 1999). Este último autor señala, además, que la permanencia de los estudiantes en la universidad dependería de un conjunto de variables determinadas por atributos de pre-ingreso, tales como: trayectoria académica en la educación secundaria, variables socioeconómicas y culturales, y metas y compromisos (Tinto, 1987). En otras palabras, tanto la literatura internacional sobre la temática (por ejemplo, Ethington, 1990, Pascarella y Terezini 1991; Slavings, Biddke y Bank, 2001; Tierney y Slaban, 1014; Jackson y Kurlaender, 2014; como recientes investigaciones nacionales (CICES, 2007; Donoso, 2010; Leyton, Vázquez y Fuenzalida, 2012), postulan que diversas habilidades o factores psicosociales pueden facilitar/obstaculizar el desarrollo de un proceso exitoso en la educación superior. Entre estas variables se cuentan la adecuación de las prácticas académicas adquiridas durante la etapa escolar a las exigencias de la educación terciaria, el capital cultural de los estudiantes y su familia, la actitud de los pares, la actitud hacia la educación superior; y la percepción de dificultad ante el estudio, expectativas de éxito y motivación del propio estudiante.

Si bien este problema es considerado un tópico de relevancia, lo que se refleja en el creciente número de investigaciones y la implementación de políticas de acceso y retención de estudiantes en la educación superior (Donoso, 2010), los hallazgos encontrados no resultan consistes respecto del peso y el sentido de los factores postulados según diferentes tipos de institución. Por ello, aún no es posible encontrar una respuesta única y significativa a esta compleja temática. 
En el año 2004, Robbins, Lauver, Le, Davis, Langley y Carlstrom, (2004), efectuaron un meta análisis, en el que examinaron la relación entre diversos factores psicosociales y los resultados académicos en la universidad. Estos autores clasificaron los factores psicosociales en 9 constructos (motivación de logro, metas académicas, compromiso institucional, apoyo social percibido, involucramiento social, autoeficacia académica, autoconcepto general, destrezas ligadas a lo académico, e influencias contextuales), los que posteriormente relacionaron con la persistencia (entendida como retención) y el rendimiento de los estudiantes. Se determinó una asociación moderada entre (i) persistencia y metas académicas, autoeficacia académica y destrezas ligadas a lo académico y (ii) entre desempeño con autoeficacia académica y motivación de logro. Luego de controlar los efectos de los predictores típicamente utilizados (vale decir, promedio general acumulado, puntajes de selección a la universidad, y nivel socioeconómico), los constructos que predecían en mayor medida la persistencia fueron: objetivos académicos, autoeficacia académica, compromiso institucional, habilidades académicas, apoyo social percibido y participación social; mientras que para el desempeño académico los mejores predictores fueron autoeficacia académica, motivación de logro y metas académicas (Robbins et al., 2004).

Conley $(2005 ; 2010)$ sugiere que el desarrollo de habilidades de preparación para la universidad se ve facilitado por el auto conocimiento del estudiante y su capacidades de planificación en torno a áreas que son clave, tales como el manejo de sus estrategias cognitivas, sus habilidades de planificación, el conocimiento del contexto y sus recursos para generar los comportamientos académicos. Algunas de estas estrategias cognitivas se refieren a comportamientos intencionales que permiten a los estudiantes aprender, entender, conservar, usar y aplicar el contenido de una amplia gama de habilidades, incluida la capacidad de hacer inferencias y deducciones lógicas a partir de su experiencia (Lombardi, Seburn y Conley, 2011).

Si bien el término preparatividad no existe en el léxico español, éste fue adoptado por los autores de la expresión inglesa preparedness, con el sentido que le fuera otorgado por Conley, (2007), como el conjunto de condiciones que cumple el estudiante para ingresar y tener éxito en una institución de educación superior. En términos prácticos, ello implica que sea capaz de aprobar los cursos básicos de manera que, sucesivamente, pueda continuar aprobando los cursos superiores, que presentan mayor dificultad. En el presente estudio el constructo preparatividad académica que, en adelante será referido como PA, no está relacionado con el dominio de aspectos específicos del currículum (que sería más apropiadamente referido como apresto), ni con haber resuelto de manera certera una opción vocacional. Específicamente, la PA incluye factores que han demostrado ser altamente gravitante en el éxito académico de los estudiantes universitarios, tales como: estrategias cognitivas, conocimiento y destrezas académicas, comportamiento académico, habilidades contextuales, autodeterminación personal, sociabilidad, modulación emocional, autoeficacia académica, anticipación analítica, comunicación efectiva y prospectiva académica (Robbins et al 2004; Tierney y Slaban, 2014).

Dado que en el contexto de la educación superior chilena, no existe un instrumento que permita medir la preparatividad académica, el objetivo del presente estudio fue construir una escala con propiedades psicométricas adecuadas, que permita evaluar la preparatividad académica que presentan las y los estudiantes que egresan de la educación secundaria y/o que recién ingresan a la educación superior.

\section{MÉTODO}

Se describe la metodología usada en este estudio en cinco subsecciones: (i) el diseño de la encuesta, (ii) los participantes en ellas, (iii) los instrumentos adicionales, (iv) el procedimiento de encuesta y (v) el análisis de los datos recolectados.

\section{Diseño}

Este estudio fue de carácter psicométrico o instrumental (Montero y León, 2005), se utilizó un diseño de encuesta transversal, ya que no existió manipulación del grado de preparatividad académica de los estudiantes y que, a través de la administración del test, se evaluó dicha variable en un momento único en el tiempo (Fontes, García-Gallego, Quintanilla, Rodríguez, Rubio y Sarriá, 2001). Lo anterior permitió posteriormente determinar la estructura y precisión de la escala desarrollada.

\section{Participantes}

Los participantes fueron seleccionados por medio de un muestreo no probabilístico intencionado o por conveniencia (Hernández, Fernández, y Baptista, 2010), por cuanto existía contacto previo con los establecimientos educacionales y universidades colaboradores. La muestra estuvo conformada por 603 participantes que cursaban entre cuarto año medio y tercer año de universidad. De ellos, el 44,1\% correspondió a hombres; el 27,5\% cursaba cuarto medio en tres establecimientos educacionales urbanos de la Región de La Araucanía, cada uno con diferente dependencia administrativa. El 40,7\% se encontraba en primer año de universidad, el $26,5 \%$ en segundo año y sólo el $5,4 \%$ en tercer año. Cabe destacar que los 
estudiantes universitarios provenían de siete instituciones ubicadas a lo largo de todo el país, cinco estatales y dos privadas. Se tuvo el propósito de reunir en la muestra a estudiantes de orígenes diferentes, por cuanto se sabe que la validez de las dimensiones emergentes de un análisis factorial depende de la fuerza de las intercorrelaciones entre las respuestas de participantes muy diversos.

\section{Instrumentos}

Cuestionario de información sociodemográfica. Con el objetivo de caracterizar a los participantes, se administró un cuestionario breve donde se solicitaba al evaluado informar su género, nombre del establecimiento educacional (colegio/universidad) y el curso/año en el que se encontraba matriculado, y la cantidad de horas semanales que dedica a estudiar. Adicionalmente, se solicitó a los estudiantes universitarios sus puntajes obtenidos en las Pruebas de Selección Universitaria (PSUß) y sus Notas en la Enseñanza Media (NEM); mientras que a los estudiantes de enseñanza secundaria se les pidió señalar las asignaturas en las que obtienen mejor rendimiento (Matemáticas, Ciencia, Lenguaje e Historia) y el promedio con el que fueron promovidos al cuarto año. Por último para determinar el nivel socioeconómico de los estudiantes, se utilizó la Escala ESOMAR (Adimark, 2000).

Escala de Preparatividad Académica para la Educación Superior (EPAES). La versión preliminar de la escala estuvo compuesta por 89 afirmaciones que reflejaban un mayor o menor grado la preparatividad académica que pudiera dar muestras un estudiante, considerando elementos afectivos, cognitivos y conativos. La mayor parte de los ítems fue elaborada por los autores, a partir del análisis de antecedentes educacionales del contexto nacional, procurando representar las dimensiones descritas en la literatura. El resto de los reactivos fue tomado de otras escalas relacionadas al constructo en estudio (ver, por ejemplo, Robbins et al., 2004; Duckworth, Peterson, Matthews, y Kelly, 2007; González, Castro y Martín, 2011). Estos ítems fueron traducidos y retraducidos por dos expertos bilingües, y los contenidos fueron descentrados para lograr que reflejaran las conductas, tal como se manifiestan en la realidad cultural de la población juvenil chilena. Todos los reactivos presentaban un continuo de cinco opciones tipo Likert para que el examinado exprese su respuesta ("en desacuerdo"; "parcialmente en desacuerdo"; "indeciso"; "parcialmente de acuerdo"; "de acuerdo"), en donde el máximo puntaje fue otorgado a las opciones de respuesta que, según la polaridad del ítem, implicaran una mayor preparatividad.

\section{Procedimiento}

Se contactó a directivos de establecimientos de educación secundaria, quienes autorizaron a acceder a sus estudiantes, quienes fueron invitados a responder la versión experimental de la escala, luego de obtenida autorización correspondiente y con la colaboración del profesor jefe de curso. Por su parte, los estudiantes universitarios fueron invitados a participar por el propio profesor de la asignatura que aceptó ceder su tiempo para la administración del instrumento. Los instrumentos fueron administrados a cada grupo curso por un integrante del equipo de investigación, y cuando ello no fue posible, se instruyó detalladamente al docente a cargo, para que administrara la escala. Previo a la distribución de los instrumento, todos los participantes recibieron un consentimiento informado, cuya firma indicaba su aceptación para participar en el estudio. En dicho texto se explicitaba el objetivo del estudio, se solicitaba la participación voluntaria y se comprometía la confidencialidad y el uso reservado de la información aportada por los participantes.

\section{Análisis de Datos}

El total de la muestra $(n=603)$ fue dividida en dos grupos, con similares características y tamaño. Con el primero de ellos se desarrolló un Análisis Factorial Exploratorio (AFE) y con la mitad restante se realizó un Análisis Factorial Confirmatorio (AFC), estrategia denominada validación cruzada. Para garantizar que no existían diferencias entre ambos grupos se realizaron pruebas estadísticas, las que permiten afirmar que ambos grupos no diferían significativamente. Inicialmente, se establecieron las evidencias de validez de contenido del test mediante la consulta a jueces expertos. Posteriormente, se evaluó las evidencias de validez de estructura del cuestionario a través de AFE, corroborándose la estructura obtenida a través de un AFC. De esta manera, para establecer si existe un adecuado grado de asociación conjunta entre los ítems para realizar los análisis propuestos se obtuvieron los estadísticos Káiser-Meyer-Olkin (KMO) y Chi cuadrado (test de Esfericidad de Bartlett). Valores de KMO superiores a .80 y un Chi-cuadrado con probabilidades inferiores a .05, respectivamente, dan cuenta de un matriz de correlaciones que permite factorización (Ferrando y Anguiano-Carrasco, 2010). Asimismo, y debido a que los datos son de naturaleza ordinal, para ejecutar el AFE se calculó el estimador WLSMV (weighted least squares means and variance adjusted) y se utilizó rotación oblicua GEOMIN. Las soluciones obtenidas fueron analizadas considerando: (i) el porcentaje de varianza explicada; (ii) su interpretabilidad teórica y (iii) la capacidad para reproducir la matriz de correlaciones, utilizando los índices de ajuste Chi cuadrado, RMSEA, CFI y TLI. En el caso de Chicuadrado, un buen ajuste está dado por probabilidades mayores a .05, mientras que para el RMSEA se ha establecido que un 'ajuste aceptable' está dado por valores entre .08 y .05, y un 'buen ajuste' por valores inferiores a .05 (Schreiber, Nora, Stage, Barlow y King, 2006). 
Asimismo, se evaluó el valor de los pesos factoriales, considerando los criterios sugeridos en Hair, Anderson, Tatham y Black (2010) para tamaños muestrales superiores a 350 personas, quienes señalan que sólo cargas factoriales superiores .30 resultan ser significativas. Finalmente, una vez establecida la estructura del cuestionario, se calculó la correlación ítem-test corregida para cada reactivo, y se estimó la confiabilidad de la escala mediante el procedimiento Alpha de Cronbach. Todos los análisis descriptivosrelacionales fueron realizados con el programa IBM SPSS versión 20.0 (IBM corp., 2011), mientras que los análisis factoriales se ejecutaron usando el programa Mplus 6.12 (Muthén y Muthén, 2006).

\section{RESULTADOS}

\section{Análisis del contenido de la escala}

Un total de siete jueces expertos, seleccionados en función de sus conocimientos en las áreas de medición e investigación educacional, respondieron una pauta de validación de contenidos, diseñada especialmente para evaluar la adecuación de los ítems formulados. En ella se definieron conceptual y operacionalmente todos los indicadores de preparatividad incluidos en la escala. De este modo, a los jueces se les solicitó evaluar (i) la claridad con que cada ítem fue redactado; (ii) su facilidad de lectura; y (iii) la representatividad de cada ítem respecto de la dimensión que orientó su construcción. Considerando las observaciones y sugerencias de los jueces, un total de 5 ítems fueron eliminados y otros 33 fueron reformulados. Así, la versión experimental del instrumento quedó compuesta por 84 ítems.

\section{Análisis de la estructura interna de la escala: AFE y AFC.}

En primer lugar se exploró la adecuación de los datos para el proceso de factorización. Tanto el estadístico de KMO (.879) como el test de Esfericidad de Bartlett $\left(\chi^{2}=7604,9 ; p<.001\right)$ dan cuenta de una matriz factorizable. A continuación, se determinó la estructura interna de la escala, desarrollándose una AFE para ello. En este procedimiento se solicitaron diferentes soluciones, donde se especificaba el número de factores a retener en función de los antecedentes teóricos existentes, contrastándose soluciones de entre cuatro a nueve factores.

Se desestimaron los modelos de cuatro y cinco factores ya que, considerando las directrices propuestas por Bentler (1990) y Tucker y Lewis (1973), los índices de ajuste incremental no alcanzaron valores dentro de los rangos aceptables (tanto CFI y TLI son inferiores a .90, lo que refleja un ajuste insuficiente). De las restantes soluciones, el modelo de siete factores resultó ser el único que admitía una interpretación teóricamente aceptable. Dicha solución exhibió niveles de ajustes aceptables (RMSEA = .029; CFI = .93; $\mathrm{TLI}=.91$ ), explicando el $59,6 \%$ de la varianza total (ver Tabla 1). Ahora, y considerando los criterios descritos en sección referida a "plan de análisis", se debieron eliminar 12 ítems en la solución retenida, ya que presentaban pesos factoriales inferiores a .30 .

Tabla 1: Índices de ajuste de las diferentes soluciones estimadas a través de AFE. ${ }^{* *} p \leq 0.01$

\begin{tabular}{|l|c|c|c|c|c|}
\hline Soluciones & $X^{2}$ & G.L. & RMSEA & CFI & TLI \\
\hline 4 Factores & $5844.03^{* *}$ & 3156 & .038 & .865 & .851 \\
\hline 5 Factores & $5240.11^{* *}$ & 3076 & .034 & .891 & .877 \\
\hline 6 Factores & $4795.41^{* *}$ & 2977 & .032 & .910 & .895 \\
\hline 7 Factores & $4394.08^{* *}$ & 2919 & .029 & .926 & .911 \\
\hline
\end{tabular}

Una vez identificada la solución exploratoria más adecuada, se evaluó la estabilidad del modelo obtenido para la medición de la preparatividad académica, con 72 ítems y siete dimensiones, efectuando un Análisis Factorial Confirmatorio (AFC). Sin embargo, dicho procedimiento arrojó niveles de ajuste inferiores a los observados en el AFE; de hecho, el estadístico Chi cuadrado resultó significativo $\left(\chi^{2}=5677.3 ; p<.001\right)$, lo que implicaba un desajuste en el modelo. Ante ello, se optó por interpretar el RMSEA, indicador de ajuste absoluto, el que sí dio cuenta de un ajuste aceptable $($ RMSEA = .051). Esta discrepancia podría ser esperable, ya que el AFC se caracteriza por ser un procedimiento altamente restrictivo, que solo permite especificar modelos en los cuales cada ítem presenta carga factorial en un determinado factor y un peso nulo en el resto de ellos (Ferrando y Anguiano-Carrasco, 2010).

Posteriormente, se calcularon las correlaciones ítem-test, corregidas, con el fin de determinar la capacidad de discriminación de cada reactivo, descartándose los ítems con valores de asociación inferiores a .30. Como resultado de este procedimiento se eliminaron cinco ítems, pertenecientes a tres dimensiones diferentes. Una vez establecida la estabilidad de modelo de 7 factores, se procedió a denominar los factores 
de acuerdo a la definición de las dimensiones actitudinales y de conducta que se entienden subyacentes a cada factor, quedando de la siguiente manera:

Factor 1: Autodeterminación Personal. Las personas que puntúan alto en esta dimensión presentan plena claridad de propósitos y la certeza de disponer de los medios y estrategias para lograrlos. Lo anterior se manifiesta también en el plano del autoconocimiento y la verbalización de sus opciones y opiniones de manera asertiva.

Factor 2: Sociabilidad. Una alta puntuación en esta dimensión es propia personas que presentan habilidades para el contacto y la comunicación social, que disfrutan y prefieren trabajar y compartir con sus pares.

Factor 3: Modulación emocional. Un alto puntaje en esta dimensión representa una capacidad para manejar de manera positiva sus reacciones emocionales y la capacidad de recuperar y/o mantener una estabilidad emocional compatible con un adecuado funcionamiento psicológico, especialmente en los ámbitos social y académico.

Factor 4: Autoeficacia académica. Una alta puntuación en esta dimensión representa una fuerte creencia de que se tiene la capacidad para alcanzar las metas académicas, con autoconocimiento y una elevada confianza en las propias capacidades. La persona se percibe capaz de persistir y esforzarse para superar las dificultades y los desafíos propios de las tareas y requerimientos académicos.

Factor 5: Anticipación analítica. Una alta puntuación en esta dimensión denota una aproximación sensible, receptiva, y reflexiva y a la vez cautelosa frente a lo que pudiera representar riesgo, tanto frente a las quehacer intelectual y académico, con frente a las relaciones interpersonales.

Factor 6: Comunicación efectiva. Una alta puntuación en esta dimensión representa una habilidad para expresar ideas de manera pertinente y organizada tanto de manera escrita como oral. Representa además una actitud positiva y autovalente respecto de tales habilidades.

Factor 7: Prospectiva Académica. Una alta puntuación indica una organización coherente y eficaz de los recursos y la generación de estrategias adecuadas, orientadas a la consecución de las metas académicas de corto, mediano y largo plazo.

Finalmente, se procedió a estimar la confiabilidad, tanto para la escala total como para las dimensiones. Tal como se aprecia en la Tabla 2, la escala total muestra un alto grado de precisión $(\alpha=.911)$. Por su parte, las dimensiones presentaron coeficientes de confiabilidad superiores a .62, lo que es aceptable.

Tabla 2:-Número de ítems e índice de confiabilidad de cada factor.

\begin{tabular}{|l|c|c|}
\hline Dimensiones y Escala total & $\mathrm{N}^{\circ}$ ítems & $\alpha$ de Cronbach \\
\hline Autodeterminación personal & 13 & .835 \\
\hline Sociabilidad & 9 & .775 \\
\hline Control y/o modulación emocional & 9 & .769 \\
\hline Autoeficacia académica & 13 & .807 \\
\hline Anticipación analítica & 6 & .623 \\
\hline Comunicación efectiva & 8 & .717 \\
\hline Prospectiva académica & 9 & .743 \\
\hline Escala Total & 67 & .914 \\
\hline
\end{tabular}

Los ítems definitivos que configuran la Escala de Preparatividad Académica para la Educación Superior (EPAES), se presentan en la Tabla 3, donde el número en paréntesis al final de cada ítem indica el factor del cual forma parte ese ítem. Además, los ítems marcados con asterisco contribuyen a medir la dimensión de manera inversa. En otras palabras, al momento de tabular los datos de la escala, será necesario invertir su puntuación.

Con los datos de la escala definitiva mostrada en la Tabla 3, se procedió a calcular los puntajes promedio por factor con todos los participantes. Una representación gráfica del perfil resultante se observa en la Figura 1. Se observa que las puntuaciones más bajas de la muestra $(n=603)$, fueron obtenidas en Control Emocional, Comunicación Efectiva y Prospectiva Académica; mientras que las puntuaciones más altas fueron obtenidas en Sociabilidad y Autoeficacia académica. 
Tabla 3: Items que componen la Escala de Preparatividad Académica para la Educación Superior (EPAES)

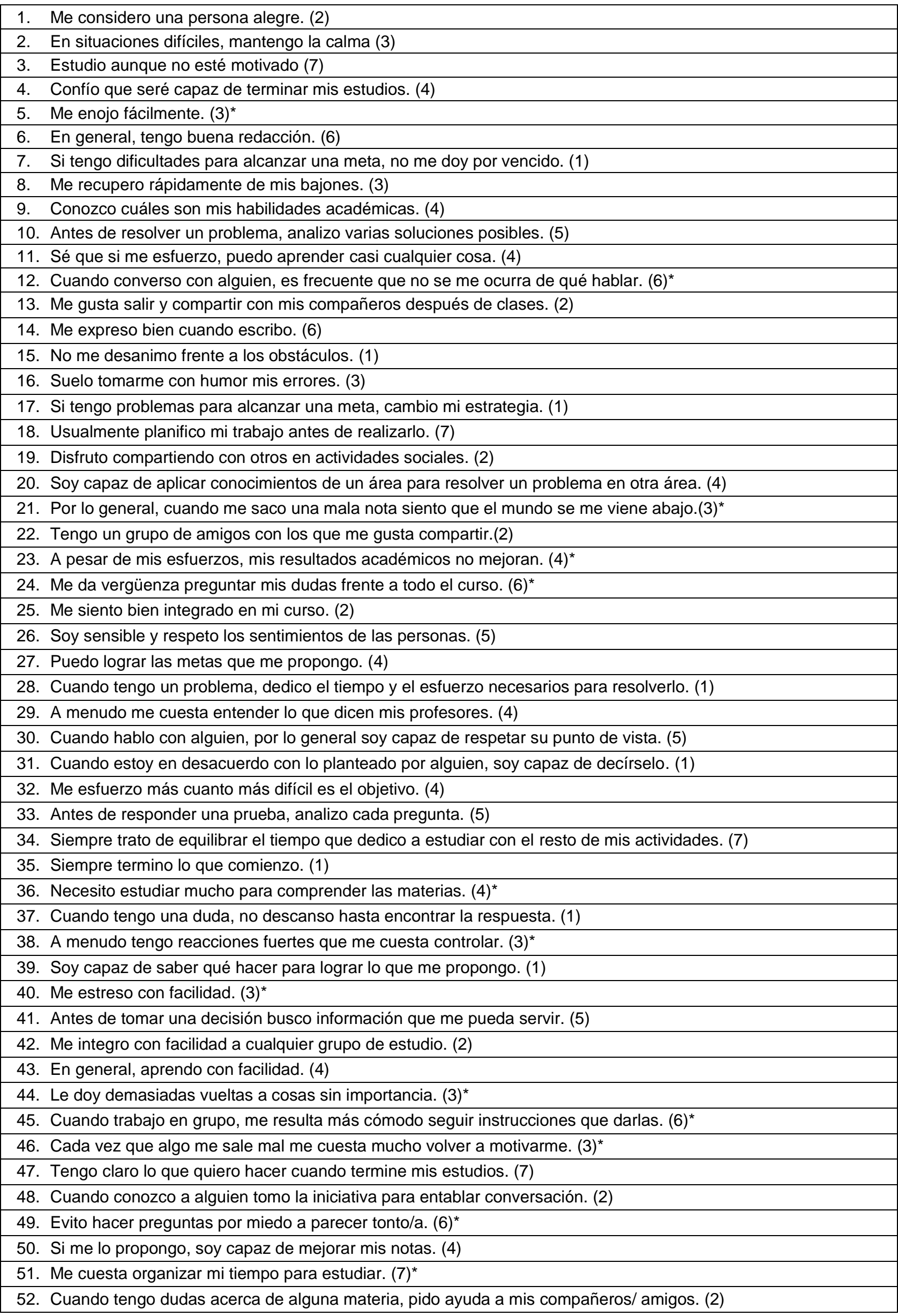


Tabla 3. Continuación

\begin{tabular}{|l|}
\hline 53. Cuando intento resolver un problema, analizo las posibles soluciones y luego escojo la mejor. (1) \\
\hline 54. Me cuesta hacer nuevos amigos. (2)* \\
\hline 55. Creo que tendré éxito en mis estudios futuros.(4) \\
\hline 56. Tengo claro cuáles son mis principales fortalezas y mis debilidades(1) \\
\hline 57. Cuando leo un texto, soy capaz de darme cuenta de qué está 'hablando' el autor. (5) \\
\hline 58. Me cuesta captar la idea principal de un texto. (4) \\
\hline 59. Tengo claras mis metas académicas. (7) \\
\hline 60. Me resulta fácil comunicar a otros mis ideas. (6) \\
\hline 61. Cuando no me va bien en una asignatura, busco alternativas para mejorar (7) \\
\hline 62. Cuando me propongo un plan, lo llevo a cabo hasta el final. (1) \\
\hline 63. Organizo mi tiempo para no estudiar a última hora. (7) \\
\hline 64. Me cuesta expresar mis ideas con precisión. (6) \\
\hline 65. Cuando me fijo mis metas, pongo todo mi esfuerzo en lograrlas. (1) \\
\hline 66. Por lo general, tomo apuntes en clases. (7) \\
\hline 67. Cuando tengo problemas, identifico fácilmente los pasos que debo seguir para resolverlos.(1) \\
\hline
\end{tabular}

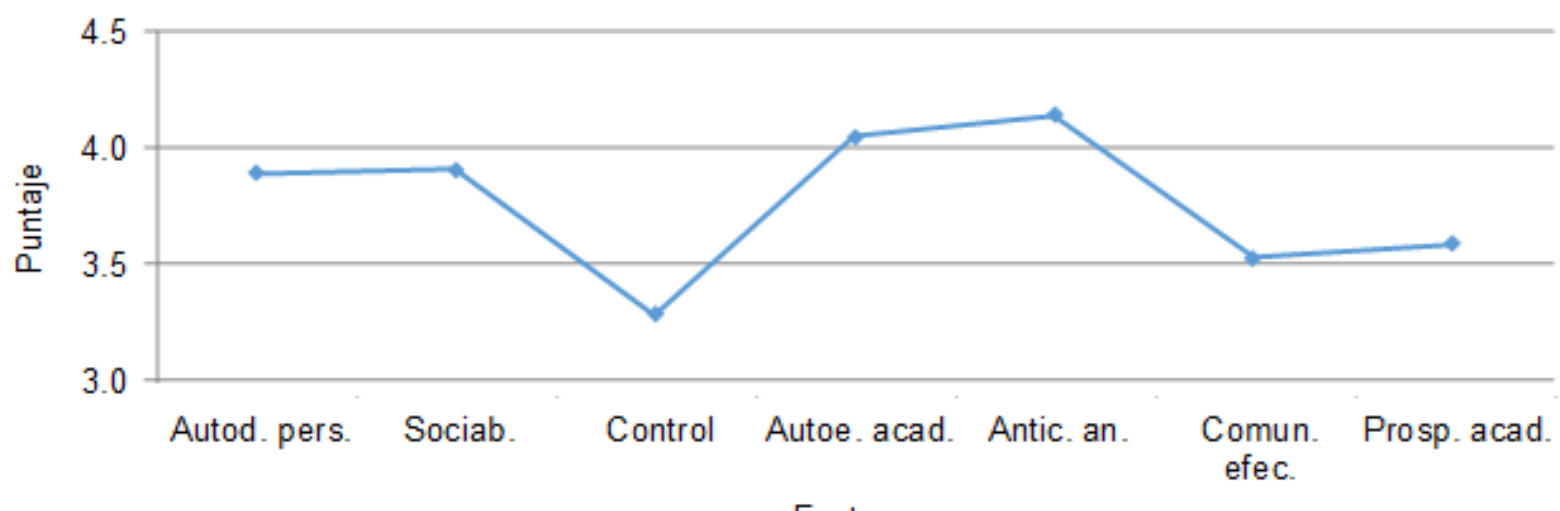

Fig. 1: Perfil de Preparatividad Académica generado con los puntajes factoriales promedio por factor para la muestra total $(n=603)$

\section{Resultados Adicionales}

En el interés de ampliar la información potencialmente rescatable de los participantes, se procedió a desagregar los datos según sexo, y se utilizó la prueba t de Student para grupos independientes, con el fin de comparar los puntajes factoriales promedio de hombres y mujeres, al interior de cada factor. Estas comparaciones mostraron que las mujeres $(n=336)$, puntuaron significativamente más alto que los hombres en las dimensiones Anticipación Analítica y Prospectiva Académica; mientras que los hombres $(n=265)$, puntuaron significativamente más alto que las mujeres en la dimensión Control Emocional $(p<0,05)$. No se observaron diferencias estadísticamente significativas entre hombres y mujeres en las demás dimensiones de la escala.

Similares comparaciones de promedios fueron efectuadas, luego de desagregar los datos según el nivel educacional de los participantes. Los resultados muestran que los estudiantes de educación media $(n=141)$ puntuaron significativamente más alto que los universitarios en Sociabilidad; mientras que los universitarios ( $\mathrm{n}=462)$, puntuaron significativamente más alto que los secundarios en Anticipación Analítica y Prospectiva Académica $(p<0,05)$. Finalmente, se buscó responder a la pregunta ies posible identificar grupos de estudiantes que presenten similitudes en sus perfiles de preparatividad académica?

Con la finalidad de responder a esta pregunta, se optó por explorar una agrupación de los sujetos a través del análisis de conglomerado jerárquico, técnica que permite - precisamente - agrupar por similitud o cercanía los puntajes factoriales de todos los participantes (Vilá, Rubio, Verlanga y Torrado, 2014). Como resultado preliminar, se obtuvo la separación de la muestra en tres conglomerados, con perfiles de preparatividad diferentes (ver gráfico de la Figura 2). 


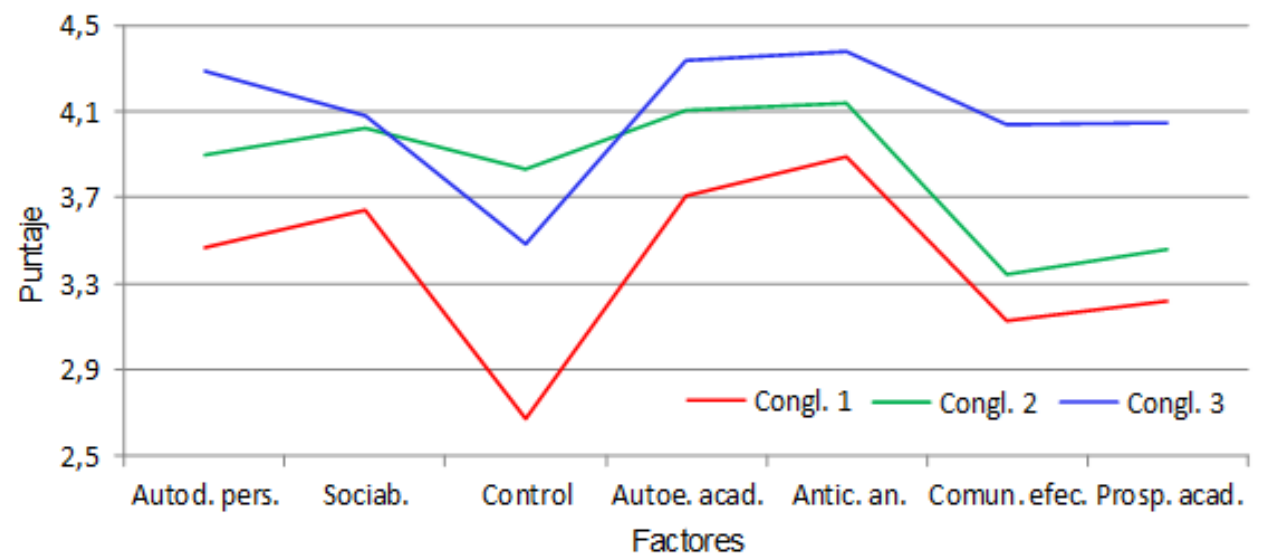

Fig. 2: Separación de la muestra en tres conglomerados de estudiantes, según la similitud, o cercanía de sus perfiles de preparatividad académica.

El conglomerado 1 agrupó al 32,6 \% de los estudiantes, mayoritariamente mujeres de nivel socioeconómico medio, quienes puntuaron sistemáticamente más bajo que los estudiantes en los otros dos conglomerados, en todos los factores, pero muestran puntuaciones especialmente deprimidas en los factores Control/modulación Emocional, Comunicación Efectiva y Prospectiva académica. Presentaban un promedio general de notas en la enseñanza media de 5,7 y un promedio PSU de 569 puntos. El conglomerado 2 agrupó al $26,8 \%$ de los estudiantes, mayoritariamente hombres que pertenecen al nivel socioeconómico medio, quienes puntuaron bastante alto en la mayoría de los factores, excepto en Comunicación Efectiva y Prospectiva académica. Además, presentaban un promedio general de notas en la enseñanza media de 5,8 y un promedio PSU de 541 puntos.

Finalmente, el conglomerado 3 agrupó al $37 \%$ de los estudiantes, mayoritariamente mujeres de nivel socioeconómico medio y medio alto, quienes puntuaron sistemáticamente más alto que los estudiantes en los otros dos conglomerados, excepto en la dimensión Control/modulación Emocional. Además, presentaban un promedio general de notas en la enseñanza media de 6,0 y un promedio PSU de 548 puntos.

\section{DISCUSIÓN}

En primer el lugar, el objetivo de construir un instrumento que permita identificar un grupo significativo de dimensiones conductuales y actitudinales que se asocian a la actividad académica de las y los estudiantes que egresan de la educación secundaria y/o que recién ingresan a la educación superior, fue plenamente logrado. En términos específicos, los análisis factoriales exploratorios y confirmatorio, permitieron seleccionar como la mejor solución un modelo de 7 factores y 67 ítemes, que explica el 59,6\% de la varianza, y cuyo índice de confiabilidad por consistencia interna de .914, es altamente satisfactorio.

Por otra parte, este estudio comprobó la existencia de un constructo o macro factor que explica una parte sustantiva de la varianza de la percepción que tienen los estudiantes acerca de sus conductas y resultados en contextos académicos; y al mismo tiempo, pone a disposición de la comunidad de investigadores y gestores educacionales, a la EPAES, como el instrumento capaz de medir de manera válida y confiable las dimensiones subyascentes a dicho constructo. Con la disponibilidad de este nuevo instrumento se podrán efectuar estudios con distintas poblaciones, desagregando datos y comparando grupos con diferentes perfiles de preparatividad, según las variables que se estime pertinente evaluar (Agudo, HernándezLunares, Rico y Sánchez, 2013). O, se podrá comparar los perfiles del mismo grupo en momentos diferentes en el tiempo, por ejemplo, antes y después de una intervención, como podría ser el caso de la evaluación del impacto de un programa de aprendizaje de habilidades sociales (Rosa, Navarro-segura y López, 2014).

Corresponde señalar también que las dimensiones que subyacen a cada uno de los factores que conforman la EPAES son muy similares a las dimensiones encontradas y descritas por Robbins y col. (2004) en su meta-análisis. Lo anterior significa que, junto con haber logrado un instrumento congruente y situado en nuestra cultura, la escala ofrece verosimilitud por cuanto presenta una configuración estructural análoga a la que presentan instrumentos elaborados en otras culturas para medir un grupo similar de variables. Sin embargo, también se observan algunas variaciones que, de seguro, reflejan diferencias culturales como podría ser la cantidad de factores encontrados, y sobre todo el surgimiento de la dimensión Sociabilidad como un componente diferenciador entre los resultados de este estudios y los efectuados con muestras de estudiantes norteamericanos (Vinet, 2010; Conley, 2010; Lombardi, Seburn y Conley, 2011). 
En cuanto a los análisis adicionales, es posible observar tres grupos diferenciados entre si los que, sin embargo, tienen una trayectoria similar en las dimensiones de la EPAES. A pesar de lo anterior, llama la atención que el conglomerado tres tenga puntuaciones más altas en la mayoría de los factores, y obtenga promedio de enseñanza media más alto que los otros dos conglomerados. Esto podría deberse a que en su mayoría contiene estudiantes que pertenecen a nivel socioeconómico medio y medio alto, es decir presentan más recursos económicos y oportunidades para su desarrollo intelectual y personal, reproduciendo las desigualdades que existen en el ámbito de la educación en Chile. Por otra parte, resulta interesante que en los tres grupos los puntajes de control emocional y comunicación efectiva sean los más bajos. Cabe preguntar si realmente la formación que se está entregando a los estudiantes va más allá de los conocimientos académicos y se está reforzando otro tipo de habilidades que permiten tener un mejor desempeño personal.

La investigación realizada entrega una herramienta útil y con adecuadas propiedades psicométricas que permite ayudar a comprender el problema de la deserción estudiantil, teniendo claridad que esta problemática seguirá existiendo en la medida que los estudiantes decidan libremente el tipo de carrera y universidad en que desean iniciar sus estudios superiores, cuando aún no tienen la madurez para ello. Por esta razón es fundamental utilizar los conocimientos empíricos derivados de instrumentos como la EPAES, de manera de disminuir el problema y los costos asociados. En la medida en que se pueda identificar el o los perfiles de preparatividad mayoritariamente asociados con deserción, será posible implementar estrategias de retención más eficientes y optimizar los recursos que se destinan al financiamiento de las mismas. De no disponer de tal información, una institución podría asignar muchos recursos para financiar programas de retención, pero los beneficios podrían ser mínimos, tanto desde la perspectiva institucional como personal de los estudiantes.

Invertir más tiempo en diagnósticos de deserción con metodologías de seguimiento adecuadas, permite mayor efectividad y minimizar costos de las acciones posteriores. Del mismo modo, la aplicación de estrategias integrales, reconociendo las causas más determinantes de la deserción en las diferentes carreras, contribuiría a una mejor relación costo-efectividad en la gestión de alumnos. Por otra parte, es importante agregar, que a pesar que las universidades hacen grandes esfuerzos por retener a sus estudiantes, sería preferible prevenir la deserción desde el momento mismo en que el alumno escoge una carrera (como un proceso preventivo más que de remedial), si se contara con los dispositivos adecuados para una medición oportuna de la preparatividad de los estudiantes. La EPAES viene ofrece ahora la posibilidad de actuar de manera preventiva.

Por otra parte, no cabe duda que la manera de percibir y manifestarse frente a algunos de los reactivos de la escala, debe estar fuertemente influenciada por la edad, ya que los estudiantes que egresan de la educación media o inician la educación superior, como es el caso de la gran mayoría de los participantes en este estudio, aún se encuentran dentro de la etapa de la adolescencia tardía, o adultez emergente (Marzana, Pérez-Acosta, Marta y González, 2010). Sin embargo, la edad puede ser considerada prácticamente una constante, en mediciones relacionadas con el ingreso a la educación terciaria.

A pesar de la robustez de los atributos de la escala recién descritos, es relevante destacar que sería interesante evaluar la validez concurrente de la EPAES. Por ejemplo, se podría considerar la utilización de la escala de autoeficacia (Sanjuán, García y Bermúdez, 2000), cuyas puntuaciones se esperaría correlacionaran positiva y significativamente con las dimensiones Autodeterminación personal y Autoeficacia académica de la EPAES. Por otra parte, una buena parte de su utilidad futura requiere analizar también la validez predictiva de la escala. Respecto a esto último, será de interés correlacionar los puntajes factoriales de la EPAES con los puntajes PSU y puntajes NEM en nuevas muestras de estudiantes. Esto último, posibilitarían la construcción de un predictor multidimensional, que combine preparatividad académica con puntajes PSU y NEM, con datos de retención y de graduación oportuna. De esta manera se puede saber si el cuestionario, permitiría identificar a aquellos estudiantes que presentan mayor riesgo y poder realizar las acciones remediales o preventivas pertinentes. De hecho, la literatura sugiere que las intervenciones adaptadas a las necesidades específicas pueden ser muy exitosas con los estudiantes en riesgo, sobre todo en materias de retención (Conley, 2010).

\section{CONCLUSIONES}

De los resultados analizados y discutidos, se puede concluir que: 1) El estudio tuvo éxito en construir un instrumento que permite medir de manera válida y confiable el grado de la preparatividad académica de estudiantes que egresan de la educación secundaria y/o que recién ingresan a la educación superior en el sistema educacional chileno. 2) La disponibilidad de este nuevo instrumento permitirá a los investigadores y gestores educacionales interesados, la medición oportuna de la preparatividad y el diseño y reorientación de acciones remediales, contribuyendo a aumentar la retención de los estudiantes y a disminuir las actuales tasas deserción universitaria en Chile. 


\section{AGRADECIMIENTOS}

Este estudio fue financiado por el Consejo Nacional de Educación, a través de la adjudicación de uno de 8 premios en el concurso de proyectos de investigación de 2012. La preparación del presente manuscrito recibió apoyo de la Dirección de Investigación de la Universidad de la Frontera, a través del proyecto EXF13-0002, y del Fondo Basal por Desempeño de la Universidad de La Frontera,Proyecto MECESUP FRO1498, 2015.

\section{REFERENCIAS}

Adimark, "El nivel socioeconómico ESOMAR". Manual de aplicación. Santiago, Chile: Autor (2000)

Agudo, J.E., Hernández-Linares, R., Rico, M y Sánchez, H., "Competencias Transversales: Percepción de su desarrollo en el Grado en Ingeniería en Diseño Industrial y Desarrollo de Productos", doi: 10.4067/S071850062013000500006, Formación Universitaria, 6(5), 39-50, (2013)

Astin, A, "Student Involvement: “A Developmental Theory for Higher Education»". Journal of College Student Personnel, 25:297-308, (1984)

Bentler, P.M, “Comparative Fit Indexes in Structural Models”. Psychological Bulletin, 107, 238-246, (1990)

Canales, A y de los Ríos, D., "Factores de la deserción universitaria", Calidad en la Educación, 26, 173-201 (2007)

Centro de Microdatos, Departamento de Economía, Universidad de Chile, "Estudios sobre causas de la deserción", Informe técnico, Santiago, Agosto, (2008)

Centro de investigación en creatividad y educación superior de la Universidad de Santiago de Chile [CICES], "Factores explicativos de la deserción universitaria, informe final", Consejo Superior de Educación (2007)

Conley, D. T, "College Knowledge: What it really takes for students to succeed and what we can do to get them ready", San Francisco, CA: Jossey-Bass, (2005)

Conley, D.T, "Toward a more comprehensive conception of college readiness", Eugene, OR: Educational. (2007)

Conley, D. T, "College and career ready: Helping all students succeed beyond high school", San Francisco, CA: Jossey-Bass, (2010)

Consejo Superior de Educación, Retención: “Algunos datos descriptivos”, Secretaría Técnica, (2008).

De Los Ríos, D, "Indicadores de calidad y eficiencia en la educación universitaria: algunas propuestas para el sistema de acreditación chileno", agosto 2000. Ed. Electrónica, disponible en: http://www.dii.uchile.cl/docencia/mgpp/EstudiosCaso/CASO44.pdf [Recuperado el día 27 de noviembre de 2013], (2000)

Donoso, S, "Retención de estudiantes y éxito académico en la educación superior: Análisis de buenas prácticas". Informe Final, Proyecto del Consejo Nacional de Educación (CNED): Santiago de Chile, (2010)

Donoso, S. y Schiefelbein, E, "Análisis de los modelos explicativos de retención de estudiantes en la universidad: Una visión desde la desigualdad social”, Estudios Pedagógicos, 33(1), 7-27, (2007)

Duckworth, A.L., Peterson, C., Matthews, M.D., \& Kelly, D.R, "Grit: Perseverance and passion for long-term goals", Journal of Personality and Social Psychology, 9, 1087-1101, (2007).

Ethington, C., "A psychological model of student persistence". Research in Higher Education. 31(31), 279$293(1990)$

Ferrando, P. J. y Anguiano-Carrasco, C., "El análisis factorial como técnica de investigación en psicología". Papeles del Psicólogo, 31(1), 18-33, (2010)

Fontes, S., García-Gallego, C., Quintanilla, L., Rodríguez, R., Rubio, P. y Sarriá, E, "Fundamentos de investigación en psicología". Madrid: UNED, (2001)

González, M., Castro, P., y Martin, M, "Personalidad Eficaz en Estudiantes Chilenos de Primer año de Ingeniería”. Formación Universitaria, 4(5), 3-12, (2011)

Hair, J. F., Anderson, R. E., Tatham, R. L. y Black, W. C, “Análisis multivariante”. Madrid, España: Pearson Prentice Hall, (2010). 
Hernández, R., Fernández, C. y Baptista, P, "Metodología de la investigación" (5a ed.). México D.F.: McGraw-Hill, (2010).

Jackson, J. y Kurlaender, M. DOI: 10.1177/0002764213515229. “College Readiness and College Completion at Board Access in Four-Year Institutions". American Behavioral Scientist, 58(8), 947-971, (2014).

IBM Corp. Released IBM SPSS Statistics for Windows, Version 20.0. Armonk, NY: IBM Corp, (2011).

Leyton, D.; Vázquez, A. y Fuenzalida, V., "La experiencia de estudiantes de contextos vulnerables en diferentes instituciones de educación superior universitaria (IESU): resultados de investigación". Revista Calidad en la Educación, 37, pp. 62-97 (2012).

Lombardi, A. Seburn, M. y Conley, D, "Development and initial validation of Academic Behaviors Associated with College and Career Readiness", Journal of Career Assessment, 19, 375-391, (2011).

Marzana, D., Pérez-Acosta, A. Marta, E. y González, I, "La transición a la edad adulta en Colombia: Una lectura Relacional". Avances en Psicología Latinoamericana, 28(1), 99-112, (2010).

Ministerio de Educación. "Informe sobre retención de Primer año de las carreras: Cohorte de Ingreso 2009. Servicio de información de la Educación Superior”, División de Educación Superior, Chile, (2010).

Montero, I. y León, O, "Sistema de clasificación del método en los informes de investigación en psicología". International Journal of Clinical and Health Psychology, 5(1), 115-127, (2005).

Muthén, L. K., y Muthén, B. O, Mplus. Los Angeles, CA: Muthén \& Muthén, (2006).

Pascarella, E., y Terenzini, P, "How college affects students: Findings and insights from twenty years of research". San Francisco: Jossey-Bass, (1991).

Rehbein, L. Baeza, M.J., y Antivilo, A, "Desarrollo y validación preliminar de una escala de preparatividad académica para la educación superior". Informe Final, Proyecto 2012, Consejo Nacional de Educación, Santiago de Chile (2013)

http://www.cned.cl/public/Secciones/Seccionlnvestigacion/investigacion_estudios_y_documentos_de_la_sectec_pdf

Robbins, S. B., Lauver, K., Le, H., Davis, D., Langley, R., y Carlstrom, A, "Do psychosocial and study skill factors predict college outcomes? A meta analysis", Psychological Bulletin, 130, 261-288, (2004).

Rosa, G., Navarro-Segura, L. y López, P, "El Aprendizaje de las Habilidades Sociales en la Universidad, Análisis de una Experiencia Formativa en los Grados de Educación Social y Trabajo Social". doi: 10.4067/S0718-50062014000400004, Formación Universitaria, 7(4), 25-38, (2014).

Sanjuán P., Pérez, A., Bermúdez, J, "Escala de autoeficacia general: datos psicométricos de la adaptación para población española". Psicothema 12(2), 509-513, (2000).

Schreiber, J. B., Nora, A., Stage, F. K., Barlow, E. A. y King, J, "Reporting Structural Equation Modeling and Confirmatory Factor Analysis Results: A Review". The Journal of Educational Research, 99(6), 323-337, (2006).

Tierney, W.G. y Slaban, J.R, “Examining College Readiness”, DOI: 10.1177/0002764213515228, American Behavioral Scientist, 58(8), 943-946, (2014).

Tinto, V, "Leaving College: Rethinking the causes and cures of student attrition", (2 ed.). Chicago: The University of Chicago Press, (1987).

Tinto, V, "Dropout from Higher Education: A theoretical synthesis of recent research". Review of Educational Research, 45: 89-125, (1975).

Tinto, V, "Taking retention seriously: Rethinking the first year of college". NACADA Journal, 19(2), 5-9, (1999).

Tucker, L. R., \& Lewis, C, "The Reliability Coefficient for Maximum Likelihood Factor Analysi"s. Psychometrika, 38, 1-10, (1973).

Vilá, R., Rubio, M.J., Verlanga, V. y Torrado, M, “Cómo aplicar un cluster jerárquico en SPSS”, DOI:10.1344/ reire2014.7.1716, REIRE, 7(1), 113-127, (2014).

Vinet, E.V, "Relativismo cultural del Modelo de Personalidad de Millon en América Latina: Un estudio con adolescentes". Interdisciplinaria, 27(1), 23-40, (2010).

Zapata, G., Tejeda, I. y Rojas, Á, "Educación Superior en Chile-Informe Nacional”. En: CINDA Educación Superior en Ibero América: Informe 2011. Centro Interuniversitario de Desarrollo. Santiago de Chile, (2011). 